\title{
Progressões aritméticas e geométricas de ordem superior e suas relações
}

\author{
Ruikson S. O. Nunes (D)
}

Juliano Sanquite Gomes (D)

\section{Resumo}

Este trabalho apresenta um breve estudo sobre Progressões Aritméticas e Geométricas, focando especialmente nas de ordem superior. São apresentadas as definições e algumas importantes propriedades dessas sequências numéricas. Um ponto importante deste trabalho é mostrar relações existentes entre Progressões Aritméticas e Geométricas de ordem k, sendo k um número natural qualquer. Mais especificamente, é mostrado que uma função de tipo exponencial transforma uma Progressão Aritmética de ordem k em uma Progressão Geométrica de ordem k e, reciprocamente, que uma função logarítmica transforma uma Progressão Geométrica de ordem k, com termos positivos, em uma Progressão Aritmética de ordem k.

Palavras-chave: Progressão Aritmética; Progressão Geométrica; Ordem Superior; Função Logarítmica; Função Exponencial.

\begin{abstract}
This paper presents a brief study on Arithmetic and Geometric Progressions, focusing especially on the higher order ones. The definitions and some important properties of these numerical sequences are presented. An important point of this work is to show the existing relations between Arithmetic and Geometric Progressions of order k, with k being a natural number. More specifically, it is shown that an exponential function transforms an Arithmetic Progression of order $\mathrm{k}$ into a Geometric Progression of order k and, conversely, that a logarithmic function transforms a Geometric Progression of order k, with positive terms, in an Arithmetic Progression of order k.
\end{abstract}

Keywords: Arithmetic Progression; Geometric Progression; Higher order; Exponential Function; Logarithmic Function.

\section{Introdução}

Muitos temas da Matemática Elementar, como a Teoria Elementar dos Números, sem dúvida alguma, são um campo muito fértil para um professor estimular e desenvolver em seus alunos o raciocínio matemático. Uma das maneiras de atingir esse propósito é estimular os alunos a fazerem, por si mesmos, pequenas generalizações ou simplesmente extensões de alguns conceitos da Matemática Elementar. Em especial, quando se trabalha com os temas progressões aritmética e geométrica, existem muitas propriedades que, com um bom estímulo, os alunos podem conseguir 
deduzir por si só. Este trabalho explora os conceitos de Progressões Aritméticas e Geométricas de ordem superior, obtendo algumas fórmulas, tais como: fórmulas para a soma e para o produto dos n primeiros termos para progressões Aritmética e Geométrica, respectivamente, e a fórmula do nésimo termo. Além disso, mostra também relações entre tais conceitos. Com isso, entendemos que o presente trabalho traz uma excelente contribuição para professores do Ensino Básico, fornecendo material e ideias que não estão presentes na maioria dos livros didáticos adotados nas escolas de Ensino Médio. Uma ideia interessante seria que, durante o ensino de Progressões Aritméticas e Geométricas, o professor motivasse os alunos a pensarem sobre a possibilidade de fazer generalizações de alguns conceitos dessas progressões, e, a partir daí, obter novas identidades matemáticas.

$\mathrm{Na}$ literatura podemos encontrar diversos trabalhos que lidam com Progressões Aritméticas e Geométricas de ordem superior fornecendo propostas de abordagens desses conceitos no Ensino Básico; para citar alguns, veja ([4], [5], [7], [8]). Nos trabalhos ([4], [7] e [8]) são estudadas apenas as Progressões Aritméticas de ordem superior e, e não se avança ao estudo das Progressões Geométricas. Já em Lopes [5], o autor faz um estudo sobre Progressões Aritméticas e Geométricas, porém, apenas para as de ordem 2.

A fim de simplificar a escrita, usaremos a sigla PA(k) para denotar Progressão Aritmética de ordem k; quando $\mathrm{k}=1$ denotaremos PA em vez de PA(1). Analogamente, Progressão Geométrica de ordem k será denotada por $\mathrm{PG}(\mathrm{k})$ e, quando $\mathrm{k}=1$, denotaremos $\mathrm{PG}$ em vez de $\mathrm{PG}(1)$.

Uma relação interessante entre uma PA e uma PG é apresentada em [3], onde é mostrado que uma função de tipo exponencial transforma uma PA em uma PG. Surge, então, uma questão interessante, que é saber se essa relação permanece válida quando abordamos progressões de ordem superior. Para a ordem 2 é mostrado em [5] que a relação permanece válida, isto é, que função de tipo exponencial transforma PA(2) em PG(2). Este trabalho avança, mostrando que tal resultado pode ser generalizado, isto é, que uma função de tipo exponencial transforma $\mathrm{PA}(\mathrm{k})$ em $\mathrm{PG}(\mathrm{k})$, para $\mathrm{k}=2,3, \cdots$. Mostraremos também que uma função logarítmica transforma uma $\mathrm{PG}(\mathrm{k})$, de termos positivos, em uma $\mathrm{PA}(\mathrm{k})$. Além disso, mostraremos algumas fórmulas básicas como: fórmula do n-ésimo termo, fórmula do produto e da soma dos n primeiros termos para $\mathrm{PG}(\mathrm{k})$ e $\mathrm{PA}(\mathrm{k})$, respectivamente. Com o propósito de melhorar o entendimento dos conceitos aqui abordados, primeiramente trabalharemos o caso particular em que $\mathrm{k}=2 \mathrm{e}$, a seguir abordamos o caso geral.

O restante deste trabalho está organizado em três seções. A Seção 2 é dedicada a expor alguns resultados e comentários preliminares que servirão de base para as seções posteriores. Na Seção 3 trabalhamos as progressões de ordem 2 e, por fim, a Seção 4 é dedicada ao estudo das progressões de ordem qualquer.

\section{Notas Preliminares}

Nesta seção faremos um breve esboço sobre PA e PG recordando algumas de suas principais propriedades. Além disso, abordaremos o conceito de função de tipo exponencial e pontuaremos algumas importantes observações sobre números binomiais que serão de fudamental importância no desenvolvimento das seções posteriores.

Definição 1. Uma sequência numérica $\left(\mathrm{x}_{1}, \mathrm{x}_{2}, \ldots, \mathrm{x}_{\mathrm{n}}, \ldots\right)$ é uma PA, quando podemos obter cada termo, a partir do segundo, como soma do termo anterior com uma constante $r$. Isto é, $x_{i+1}=x_{i}+r$ para todo $i=1,2, \cdots$. A constante $r$ é chamada de razão da PA.

Exemplo 1. A sequência $(3,5,7, \ldots, 2 \mathrm{n}+1, \ldots)$ é uma PA de razão $\mathrm{r}=2$, pois cada termo, a partir do segundo, é obtido somando o termo anterior com a constante 2. 
As fórmulas de recorrência que determinam o n-ésimo termo de uma PA de razão r e a soma $S_{n}$ de seus n-primeiros termos são dadas, respectivamente, por;

$$
\begin{gathered}
x_{n}=x_{1}+(n-1) r \\
S_{n}=n_{1}+\frac{n(n-1)}{2} r .
\end{gathered}
$$

Definição 2. Uma sequência numérica $\left(\mathrm{x}_{1}, \mathrm{x}_{2}, \cdots, \mathrm{x}_{\mathrm{n}}, \ldots\right)$ é uma PG, quando podemos obter cada termo, a partir do segundo, como produto do termo anterior com uma constante q. Isto é, $\mathrm{x}_{\mathrm{i}+1}=$ $\mathrm{x}_{\mathrm{i}} \cdot \mathrm{q}$ para todo $\mathrm{i}=1,2, \cdots$. A constante $\mathrm{q}$ é chamada de razão da $\mathrm{PG}$.

Exemplo 2. A sequência $\left(3,6,12, \ldots, 3 \cdot 2^{n-1}, \ldots\right)$ é uma $P G$ de razão $q=2$, pois cada termo, a partir do segundo, é obtido multiplicando o termo anterior com a constante 2.

Como pode ser visto em [6], as fórmulas de recorrência que determinam o n-ésimo termo de uma $\mathrm{PG}$ de razão $\mathrm{q}$, bem como o produto $\mathrm{P}_{\mathrm{n}}$ dos seus $\mathrm{n}$ primeiros termos, são dadas, respectivamente, por

$$
\begin{gathered}
x_{n}=x_{1} q^{n-1}, \\
P_{n}=x_{1}^{n} q^{\frac{n(n-1)}{2}} .
\end{gathered}
$$

Consideremos a e $\mathrm{b}$ números reais positivos com $\mathrm{a} \neq 1$. A função $\mathrm{f}: \mathbb{R} \rightarrow \mathbb{R}$ definida por $\mathrm{f}(\mathrm{x})=\mathrm{ba}^{\mathrm{x}}$ é chamada tipo exponencial. O Teorema abaixo, encontrado em [3], mostra como PA e PG relacionam-se por meio de uma função de tipo exponencial.

Teorema 1. Sejam $\left(\mathrm{x}_{1}, \mathrm{x}_{2}, \ldots, \mathrm{x}_{\mathrm{n}}, \ldots\right)$ uma PA de razão $\mathrm{r}$ e $\mathrm{f}(\mathrm{x})=\mathrm{ba}^{\mathrm{x}}$ uma função de tipo exponencial. Então, a sequência $\left(\mathrm{f}\left(\mathrm{x}_{1}\right), \mathrm{f}\left(\mathrm{x}_{2}\right), \ldots, \mathrm{f}\left(\mathrm{x}_{\mathrm{n}}\right), \ldots\right.$ ) é uma $P G$ de razão $\mathrm{a}^{\mathrm{r}}$.

Demonstração. Como $\mathrm{r}$ é a razão da PA $\left(\mathrm{x}_{1}, \mathrm{x}_{2}, \ldots, \mathrm{x}_{\mathrm{n}}, \ldots\right)$, segue que $\mathrm{r}=\mathrm{x}_{\mathrm{n}+1}-\mathrm{x}_{\mathrm{n}}$ para todo número natural $n$. Por outro lado, note que $\frac{f\left(x_{n+1}\right)}{f\left(x_{n}\right)}=\frac{a^{x_{n+1}}}{a^{x_{n}}}=a^{r}$ para todo $n$ natural, garantindo que a sequência $\left(f\left(x_{1}\right), f\left(x_{2}\right), \ldots, f\left(x_{n}\right), \ldots\right)$ é uma $P G$.

A fim de obter as fórmulas gerais para progressões aritméticas e geométricas de ordem superior serão necessárias algumas propriedades dos números binomiais. Existem, na literatura, várias obras que tratam desse assunto, veja ([6], [1], [2]).

Considerando $\mathrm{m}$ e $\mathrm{n}$ números inteiros não negativos, o número binomial $\left(\begin{array}{l}\mathrm{n} \\ \mathrm{m}\end{array}\right)$ é definido, conforme $[2]$, por

$$
\left(\begin{array}{l}
n \\
m
\end{array}\right)= \begin{cases}\frac{n !}{m !(n-m) !}, & \text { se } n \geqslant m \\
0, & \text { se } n<m\end{cases}
$$

O número n representa a linha e m a coluna onde o número está posicionado no Triângulo de Pascal. Um resultado que terá papel importante neste trabalho é o chamado Teorema das Colunas, exposto no Lema 1 abaixo. 
Lema 1. (Teorema das Colunas) Dados inteiros não negativos $\mathrm{n}$ e $\mathrm{p}$, a soma dos $\mathrm{p}+1$ primeiros números da coluna $\mathrm{n}$ do Triângulo de Pascal é:

$$
\left(\begin{array}{l}
\mathrm{n} \\
\mathrm{n}
\end{array}\right)+\left(\begin{array}{c}
\mathrm{n}+1 \\
\mathrm{n}
\end{array}\right)+\cdots+\left(\begin{array}{c}
\mathrm{n}+\mathrm{p} \\
\mathrm{n}
\end{array}\right)=\left(\begin{array}{c}
\mathrm{n}+\mathrm{p}+1 \\
\mathrm{n}+1
\end{array}\right) .
$$

Uma demonstração do Lema 1 pode ser vista em [1].

\section{Progressões Aritméticas e Geométricas de $2^{\text {a }}$ ordem}

Definição 3. Uma sequência $\left(\mathrm{x}_{1}, \mathrm{x}_{2}, \ldots, \mathrm{x}_{\mathrm{n}}, \ldots\right)$ é uma Progressão Aritmética de segunda ordem, isto é, $\mathrm{PA}(2)$, quando a sequência de diferenças $\left(\mathrm{d}_{1}, \mathrm{~d}_{2}, \ldots, \mathrm{d}_{\mathrm{n}}, \ldots\right)$, com $\mathrm{d}_{\mathrm{n}}=\mathrm{x}_{\mathrm{n}+1}-\mathrm{x}_{\mathrm{n}}$, for uma PA.

Exemplo 3. A sequência $(1,3,7,13,21, \ldots, 1+n(n-1), \ldots)$ é uma $P A(2)$, pois a sequência de diferenças $(2,4,6,8, \ldots, 2 \mathrm{n}, \ldots)$ é uma PA de razão igual a 2 .

Para deduzir a fórmula do n-ésimo termo de uma $\mathrm{PA}(2)$, digamos que a sequência $\left(\mathrm{d}_{1}, \mathrm{~d}_{2}, \ldots, \mathrm{d}_{\mathrm{n}}, \cdots\right)$ seja uma PA de razão r. Assim, temos:

$$
\begin{aligned}
\mathrm{x}_{\mathrm{n}} & =\mathrm{x}_{1}+\left(\mathrm{d}_{1}+\mathrm{d}_{2}+\cdots+\mathrm{d}_{\mathrm{n}-1}\right) \\
& =\mathrm{x}_{1}+(\mathrm{n}-1) \mathrm{d}_{1}+\frac{(\mathrm{n}-1)(\mathrm{n}-2)}{2} \mathrm{r} \\
& =\left(\begin{array}{c}
\mathrm{n}-1 \\
0
\end{array}\right) \mathrm{x}_{1}+\left(\begin{array}{c}
\mathrm{n}-1 \\
1
\end{array}\right) \mathrm{d}_{1}+\left(\begin{array}{c}
\mathrm{n}-1 \\
2
\end{array}\right) \mathrm{r} .
\end{aligned}
$$

Na segunda igualdade acima, usamos a fórmula (2) para somar os termos da PA $\left(\mathrm{d}_{1}, \mathrm{~d}_{2}, \ldots, \mathrm{d}_{\mathrm{n}}, \cdots\right)$ e o fato de que $\mathrm{x}_{1}=\left(\begin{array}{c}\mathrm{n}-1 \\ 0\end{array}\right) \mathrm{x}_{1}$.

Usando o Lema 1, obtemos a fórmula da soma dos n primeiros termos da $\mathrm{PA}(2)$ :

$$
\begin{aligned}
\sum_{\mathrm{i}=1}^{\mathrm{n}} \mathrm{x}_{\mathrm{i}} & =\sum_{\mathrm{i}=1}^{\mathrm{n}}\left(\begin{array}{c}
\mathrm{i}-1 \\
0
\end{array}\right) \mathrm{x}_{1}+\sum_{\mathrm{i}=1}^{\mathrm{n}}\left(\begin{array}{c}
\mathrm{i}-1 \\
1
\end{array}\right) \mathrm{d}_{1}+\sum_{\mathrm{i}=1}^{\mathrm{n}}\left(\begin{array}{c}
\mathrm{i}-1 \\
2
\end{array}\right) \mathrm{r} \\
& =\left(\begin{array}{l}
\mathrm{n} \\
1
\end{array}\right) \mathrm{x}_{1}+\left(\begin{array}{l}
\mathrm{n} \\
2
\end{array}\right) \mathrm{d}_{1}+\left(\begin{array}{l}
\mathrm{n} \\
3
\end{array}\right) \mathrm{r} .
\end{aligned}
$$

Observação 1. Levando em conta a definição de número binomial concluímos que $\sum_{i=1}^{n}\left(\begin{array}{c}i-1 \\ j\end{array}\right)=\sum_{i=j+1}^{n}\left(\begin{array}{c}i-1 \\ j\end{array}\right)$. Assim, na fórmula (6), temos especificamente, $\sum_{i=1}^{n}\left(\begin{array}{c}i-1 \\ 1\end{array}\right)=\sum_{i=2}^{n}\left(\begin{array}{c}i-1 \\ 1\end{array}\right)$ e $\sum_{i=1}^{n}\left(\begin{array}{c}i-1 \\ 1\end{array}\right)=\sum_{i=3}^{n}\left(\begin{array}{c}i-1 \\ 2\end{array}\right)$.

Trataremos, a seguir, o conceito de Progressão Geométrica de segunda ordem, ou PG(2).

Definição 4. Uma sequência $\left(\mathrm{x}_{1}, \mathrm{x}_{2}, \ldots, \mathrm{x}_{\mathrm{n}}, \ldots\right)$ é uma Progressão Geométrica de segunda ordem, isto é PG(2), quando a sequência de quocientes $\left(q_{1}, q_{2}, \ldots, q_{n}, \ldots\right)$, com $q_{n}=\frac{x_{n+1}}{x_{n}}$, for uma PG.

Exemplo 4. A sequência $\left(1,3,18,216, \cdots, 3^{(\mathrm{n}-1)} \cdot 2^{\frac{(\mathrm{n}-1)(\mathrm{n}-2)}{2}}, \ldots\right)$ é uma $\mathrm{PG}(2)$, pois a sequência de quocientes $\left(3,6,12, \ldots, 3 \cdot 2^{(n-1)}, \ldots\right)$ é uma $\mathrm{PG}$ de razão igual a 2 . 
Primeiramente determinaremos a fórmula do termo geral de uma PG(2) $\left(\mathrm{x}_{1}, \mathrm{x}_{2}, \ldots, \mathrm{x}_{\mathrm{n}}, \ldots\right)$ cuja sequência de quocientes $\left(q_{1}, q_{2}, \ldots, q_{n}, \cdots\right)$ é uma $P G$ de razão q. Veja que

$$
\begin{aligned}
x_{n} & =x_{1} \cdot q_{1} \cdot q_{2} \cdots q_{n-1} \\
& =x_{1} \cdot q_{1}^{(n-1)} \cdot q^{\frac{(n-1)(n-2)}{2}} \\
& =x_{1}^{\left(\begin{array}{c}
n-1 \\
0
\end{array}\right)} \cdot q_{1}^{\left(\begin{array}{c}
n-1 \\
1
\end{array}\right)} \cdot q^{\left(\begin{array}{c}
n-1 \\
2
\end{array}\right)} .
\end{aligned}
$$

Na segunda equação acima usamos a fórmula do produto dos termos de uma PG, estabelecido em (4).

Agora, considerando o Lema 1 e a Observação 1, obtemos a fórmula do produto dos n primeiros termos de uma $\mathrm{PG}(2)$ :

$$
\begin{aligned}
\prod_{i=1}^{n} x_{i} & =x_{1}^{\sum_{i=1}^{n}\left(\begin{array}{c}
\mathrm{i}-1 \\
0
\end{array}\right)} \cdot q_{1}^{\sum_{i=1}^{n}\left(\begin{array}{c}
\mathrm{i}-1 \\
1
\end{array}\right)} \cdot q^{\sum_{\mathrm{i}=2}^{\mathrm{n}}\left(\begin{array}{c}
\mathrm{i}-1 \\
2
\end{array}\right)} \\
& =\mathrm{x}_{1}^{\left(\begin{array}{c}
\mathrm{n} \\
1
\end{array}\right)} \cdot \mathrm{q}_{1}^{\left(\begin{array}{c}
\mathrm{n} \\
2
\end{array}\right)} \cdot \mathrm{q}^{\left(\begin{array}{c}
\mathrm{n} \\
3
\end{array}\right)} .
\end{aligned}
$$

Para finalizar esta seção mostraremos que a função de tipo exponencial $f(x)=b a^{x}$, definida anteriormente, transforma $\mathrm{PA}(2)$ em $\mathrm{PG}(2)$.

Teorema 2. Sejam $\left(\mathrm{x}_{1}, \mathrm{x}_{2}, \ldots, \mathrm{x}_{\mathrm{n}}, \ldots\right)$ uma PA(2) e $\mathrm{f}(\mathrm{x})=\mathrm{ba}^{\mathrm{x}}$ uma função do tipo exponencial. Então, a sequência $\left(\mathrm{f}\left(\mathrm{x}_{1}\right), \mathrm{f}\left(\mathrm{x}_{2}\right), \ldots, \mathrm{f}\left(\mathrm{x}_{\mathrm{n}}\right), \ldots\right)$ é uma $P G(2)$.

Demonstração. Sendo $\left(\mathrm{x}_{1}, \mathrm{x}_{2}, \cdots, \mathrm{x}_{\mathrm{n}}, \ldots\right)$ uma $\mathrm{PA}(2)$, então a sequência de diferenças $\left(\mathrm{d}_{1}, \mathrm{~d}_{2}, \ldots, \mathrm{d}_{\mathrm{n}}, \ldots\right)$, onde $\mathrm{d}_{\mathrm{n}}=\mathrm{x}_{\mathrm{n}+1}-\mathrm{x}_{\mathrm{n}}$, é uma PA, e suponhamos que sua razão seja r. Considerando a função exponencial $g(x)=a^{x}$, pelo Teorema 1 , segue que $\left(a^{d_{1}}, a^{d_{2}}, \ldots, a^{d_{n}}, \ldots\right)$ é uma PG de razão $a^{r}$. Agora, observe que $\frac{f\left(x_{n+1}\right)}{f\left(x_{n}\right)}=a^{\left(x_{n+1}-x_{n}\right)}=a^{d_{n}}$, para todo $n$ natural. Logo, concluímos que $\left(\mathrm{f}\left(\mathrm{x}_{1}\right), \mathrm{f}\left(\mathrm{x}_{2}\right), \ldots, \mathrm{f}\left(\mathrm{x}_{\mathrm{n}}\right), \ldots\right)$ é uma $\mathrm{PG}(2)$.

\section{Progressões Aritméticas e Geométricas de ordem k}

Seja k um número inteiro não negativo. Afim definir Progressões Aritméticas de ordem k, definiremos o conceito de operador diferença de ordem $\mathrm{k}$.

Definição 5. Seja $\left(\mathrm{x}_{1}, \mathrm{x}_{2}, \ldots, \mathrm{x}_{\mathrm{n}}, \ldots\right)$ uma sequência de números reais. $\mathrm{O}$ operador diferença de ordem $\mathrm{k}$, denotado por $\mathrm{D}^{\mathrm{k}}$, é definido recursivamente por $\mathrm{D}^{\mathrm{k}} \mathrm{x}_{\mathrm{n}}=\mathrm{D}^{1}\left(\mathrm{D}^{\mathrm{k}-1} \mathrm{x}_{\mathrm{n}}\right)=\mathrm{D}^{\mathrm{k}-1} \mathrm{x}_{\mathrm{n}+1}-\mathrm{D}^{\mathrm{k}-1} \mathrm{x}_{\mathrm{n}}$.

Pela forma em que foi definido, note que o operador diferença $D^{k}$ satisfaz $D^{j}\left(D^{k} x_{n}\right)=D^{j+k} x_{n}$. Por exemplo, $\mathrm{D}^{2} \mathrm{x}_{\mathrm{n}}=\mathrm{D}^{1}\left(\mathrm{D}^{1} \mathrm{x}_{\mathrm{n}}\right)=\mathrm{D}^{1} \mathrm{x}_{\mathrm{n}+1}-\mathrm{D}^{1} \mathrm{x}_{\mathrm{n}}, \mathrm{D}^{3} \mathrm{x}_{\mathrm{n}}=\mathrm{D}^{1}\left(\mathrm{D}^{2} \mathrm{x}_{\mathrm{n}}\right)=\mathrm{D}^{2} \mathrm{x}_{\mathrm{n}+1}-\mathrm{D}^{2} \mathrm{x}_{\mathrm{n}}$, e assim sucessivamente.

Definição 6. Uma sequência $\left(\mathrm{x}_{1}, \mathrm{x}_{2}, \ldots, \mathrm{x}_{\mathrm{n}}, \ldots\right)$ é uma Progressão Aritmética de ordem $\mathrm{k}>1$, isto é, $\mathrm{PA}(\mathrm{k})$, quando a sequência de diferenças $\left(\mathrm{D}^{1} \mathrm{x}_{1}, \mathrm{D}^{1} \mathrm{x}_{2}, \ldots, \mathrm{D}^{1} \mathrm{x}_{\mathrm{n}}, \ldots\right), \operatorname{com} \mathrm{D}^{1} \mathrm{x}_{\mathrm{n}}=\mathrm{x}_{\mathrm{n}+1}-\mathrm{x}_{\mathrm{n}}$, for uma $\mathrm{PA}(\mathrm{k}-1)$.

A fim de simplificar notação, vamos fixar o seguinte; $\mathrm{r}_{0}=\mathrm{D}^{0} \mathrm{x}_{1}, \mathrm{r}_{1}=\mathrm{D}^{1} \mathrm{x}_{1}, \mathrm{r}_{2}=\mathrm{D}^{2} \mathrm{x}_{1}, \cdots, \mathrm{r}_{\mathrm{k}}=\mathrm{D}^{\mathrm{k}} \mathrm{x}_{1}$. Veja que $r_{0}$ é o primeiro termo da sequência original, $r_{1}$ é o primeiro termo da sequência de 
diferenças de ordem 1; enfim, $\mathrm{r}_{\mathrm{k}}$ é o primeiro termo da sequência de diferenças de ordem $\mathrm{k}$. A fórmula do n-ésimo termo e a da soma dos n primeiros termos de uma PA(k) são estabelecidas no seguinte teorema.

Teorema 3. Seja $\left(\mathrm{x}_{1}, \mathrm{x}_{2}, \ldots, \mathrm{x}_{\mathrm{n}}, \ldots\right)$ é uma Progressão Aritmética de ordem $\mathrm{k}>1$. O $\mathrm{n}$-ésimo termo é determinado por;

$$
\mathrm{x}_{\mathrm{n}}=\left(\begin{array}{c}
\mathrm{n}-1 \\
0
\end{array}\right) \mathrm{r}_{0}+\left(\begin{array}{c}
\mathrm{n}-1 \\
1
\end{array}\right) \mathrm{r}_{1}+\left(\begin{array}{c}
\mathrm{n}-1 \\
2
\end{array}\right) \mathrm{r}_{2}+\cdots+\left(\begin{array}{c}
\mathrm{n}-1 \\
\mathrm{k}
\end{array}\right) \mathrm{r}_{\mathrm{k}}
$$

e a soma de seus n primeiros termos é determinada por;

$$
\sum_{\mathrm{i}=1}^{\mathrm{n}} \mathrm{x}_{\mathrm{i}}=\left(\begin{array}{l}
\mathrm{n} \\
1
\end{array}\right) \mathrm{r}_{0}+\left(\begin{array}{l}
\mathrm{n} \\
2
\end{array}\right) \mathrm{r}_{1}+\left(\begin{array}{l}
\mathrm{n} \\
3
\end{array}\right) \mathrm{r}_{2}+\cdots+\left(\begin{array}{c}
\mathrm{n} \\
\mathrm{k}+1
\end{array}\right) \mathrm{r}_{\mathrm{k}} .
$$

Demonstração. Provaremos primeiramente a fórmula do n-ésimo termo usando a indução sobre k. Se $\mathrm{k}=2$, temos

$$
\mathrm{x}_{\mathrm{n}}=\left(\begin{array}{c}
\mathrm{n}-1 \\
0
\end{array}\right) \mathrm{r}_{0}+\left(\begin{array}{c}
\mathrm{n}-1 \\
1
\end{array}\right) \mathrm{r}_{1}+\left(\begin{array}{c}
\mathrm{n}-1 \\
2
\end{array}\right) \mathrm{r}_{2},
$$

que é o n-ésimo termo de uma PA(2), conforme a igualdade (5). Por hipótese, suponhamos que a fórmula (9) seja válida para uma $\mathrm{PA}(\mathrm{k}-1)$. Sendo $\left(\mathrm{x}_{1}, \mathrm{x}_{2}, \cdots, \mathrm{x}_{\mathrm{n}}, \cdots\right)$ uma $\mathrm{PA}(\mathrm{k})$, segue que a sequência de diferenças $\left(\mathrm{D}^{1} \mathrm{x}_{1}, \mathrm{D}^{1} \mathrm{x}_{2}, \cdots, \mathrm{D}^{1} \mathrm{x}_{\mathrm{n}}, \cdots\right)$ é uma $\mathrm{PA}(\mathrm{k}-1)$. Assim, pela hipótese de indução segue que

$$
\mathrm{D}^{1} \mathrm{x}_{\mathrm{n}}=\left(\begin{array}{c}
\mathrm{n}-1 \\
0
\end{array}\right) \mathrm{D}^{1} \mathrm{x}_{1}+\left(\begin{array}{c}
\mathrm{n}-1 \\
1
\end{array}\right) \mathrm{D}^{2} \mathrm{x}_{1}+\left(\begin{array}{c}
\mathrm{n}-1 \\
2
\end{array}\right) \mathrm{D}^{3} \mathrm{x}_{1}+\cdots+\left(\begin{array}{l}
\mathrm{n}-1 \\
\mathrm{k}-1
\end{array}\right) \mathrm{D}^{\mathrm{k}} \mathrm{x}_{1}
$$

Agora, levando em conta o Lema 1, a Observação 1, bem como essa ultima igualdade, obtemos;

$$
\begin{aligned}
\mathrm{x}_{\mathrm{n}} & =\mathrm{x}_{1}+\left(\mathrm{x}_{2}-\mathrm{x}_{1}\right)+\left(\mathrm{x}_{3}-\mathrm{x}_{2}\right)+\left(\mathrm{x}_{4}-\mathrm{x}_{3}\right)+\cdots+\left(\mathrm{x}_{\mathrm{n}}-\mathrm{x}_{\mathrm{n}-1}\right) \\
& =\mathrm{x}_{1}+\left(\mathrm{D}^{1} \mathrm{x}_{1}+\mathrm{D}^{1} \mathrm{x}_{2}+\mathrm{D}^{1} \mathrm{x}_{3}+\cdots+\mathrm{D}^{1} \mathrm{x}_{\mathrm{n}-1}\right) \\
& =\mathrm{x}_{1}+\sum_{\mathrm{i}=1}^{\mathrm{n}-1} \mathrm{D}^{1} \mathrm{x}_{\mathrm{i}} \\
& =\mathrm{x}_{1}+\sum_{\mathrm{i}=1}^{\mathrm{n}-1}\left(\begin{array}{c}
\mathrm{i}-1 \\
0
\end{array}\right) \mathrm{D}^{1} \mathrm{x}_{1}+\sum_{\mathrm{i}=1}^{\mathrm{n}-1}\left(\begin{array}{c}
\mathrm{i}-1 \\
1
\end{array}\right) \mathrm{D}^{2} \mathrm{x}_{1}+\sum_{\mathrm{i}=1}^{\mathrm{n}-1}\left(\begin{array}{c}
\mathrm{i}-1 \\
2
\end{array}\right) \mathrm{D}^{3} \mathrm{x}_{1}+\cdots+\sum_{\mathrm{i}=1}^{\mathrm{n}-1}\left(\begin{array}{c}
\mathrm{i}-1 \\
\mathrm{k}-1
\end{array}\right) \mathrm{D}^{\mathrm{k}} \mathrm{x}_{1} \\
& =\mathrm{D}^{0} \mathrm{x}_{1}+\left(\begin{array}{c}
\mathrm{n}-1 \\
1
\end{array}\right) \mathrm{D}^{1} \mathrm{x}_{1}+\left(\begin{array}{c}
\mathrm{n}-1 \\
2
\end{array}\right) \mathrm{D}^{2} \mathrm{x}_{1}+\left(\begin{array}{c}
\mathrm{n}-1 \\
3
\end{array}\right) \mathrm{D}^{3} \mathrm{x}_{1}+\cdots+\left(\begin{array}{c}
\mathrm{n}-1 \\
\mathrm{k}
\end{array}\right) \mathrm{D}^{\mathrm{k}} \mathrm{x}_{1} \\
& =\left(\begin{array}{c}
\mathrm{n}-1 \\
0
\end{array}\right) \mathrm{r}_{0}+\left(\begin{array}{c}
\mathrm{n}-1 \\
1
\end{array}\right) \mathrm{r}_{1}+\left(\begin{array}{c}
\mathrm{n}-1 \\
2
\end{array}\right) \mathrm{r}_{2}+\cdots+\left(\begin{array}{c}
\mathrm{n}-1 \\
\mathrm{k}
\end{array}\right) \mathrm{r}_{\mathrm{k}} .
\end{aligned}
$$

Para provarmos a fórmula (10) utilizamos a fórmula (9), o Lema 1 e a Observação 1; assim obtemos;

$$
\begin{aligned}
\sum_{\mathrm{i}=1}^{\mathrm{n}} \mathrm{x}_{\mathrm{i}} & =\sum_{\mathrm{i}=1}^{\mathrm{n}}\left(\begin{array}{c}
\mathrm{i}-1 \\
0
\end{array}\right) \mathrm{r}_{0}+\sum_{\mathrm{i}=1}^{\mathrm{n}}\left(\begin{array}{c}
\mathrm{i}-1 \\
1
\end{array}\right) \mathrm{r}_{1}+\sum_{\mathrm{i}=1}^{\mathrm{n}}\left(\begin{array}{c}
\mathrm{i}-1 \\
2
\end{array}\right) \mathrm{r}_{2}+\cdots+\sum_{\mathrm{i}=1}^{\mathrm{n}}\left(\begin{array}{c}
\mathrm{i}-1 \\
\mathrm{k}
\end{array}\right) \mathrm{r}_{\mathrm{k}} . \\
& =\left(\begin{array}{c}
\mathrm{n} \\
1
\end{array}\right) \mathrm{r}_{0}+\left(\begin{array}{l}
\mathrm{n} \\
2
\end{array}\right) \mathrm{r}_{1}+\left(\begin{array}{l}
\mathrm{n} \\
3
\end{array}\right) \mathrm{r}_{2}+\cdots+\left(\begin{array}{c}
\mathrm{k} \\
\mathrm{k}+1
\end{array}\right) \mathrm{r}_{\mathrm{k}} .
\end{aligned}
$$


A seguir definiremos Progressão Geométrica de ordem $\mathrm{k}>1$. Para isso, definiremos operador quociente de ordem $\mathrm{k}$, que é dado recursivamente por $\mathrm{Q}^{\mathrm{k}} \mathrm{x}_{\mathrm{n}}=\mathrm{Q}^{1}\left(\mathrm{Q}^{\mathrm{k}-1} \mathrm{x}_{\mathrm{n}}\right)=\frac{\mathrm{Q}^{\mathrm{k}-1} \mathrm{x}_{\mathrm{n}+1}}{\mathrm{Q}^{\mathrm{k}-1} \mathrm{x}_{\mathrm{n}}}$. Por exemplo, $\mathrm{Q}^{2} \mathrm{x}_{\mathrm{n}}=\mathrm{Q}^{1}\left(\mathrm{Q}^{1} \mathrm{x}_{\mathrm{n}}\right)=\frac{\mathrm{Q}^{1} \mathrm{x}_{\mathrm{n}+1}}{\mathrm{Q}^{1} \mathrm{x}_{\mathrm{n}}}, \mathrm{Q}^{3} \mathrm{x}_{\mathrm{n}}=\mathrm{Q}^{1}\left(\mathrm{Q}^{2} \mathrm{x}_{\mathrm{n}}\right)=\frac{\mathrm{Q}^{2} \mathrm{x}_{\mathrm{n}+1}}{\mathrm{Q}^{2} \mathrm{x}_{\mathrm{n}}}$, e assim sucessivamente.

Definição 7. Uma sequência $\left(\mathrm{x}_{1}, \mathrm{x}_{2}, \ldots, \mathrm{x}_{\mathrm{n}}, \ldots\right)$ é uma Progressão Geométrica de ordem $\mathrm{k}$, isto é, $\mathrm{PG}(\mathrm{k})$, quando a sequência de quocientes $\left(\mathrm{Q}^{1} \mathrm{x}_{1}, \mathrm{Q}^{1} \mathrm{x}_{2}, \ldots, \mathrm{Q}^{1} \mathrm{x}_{\mathrm{n}}, \ldots\right)$, com $\mathrm{Q}^{1} \mathrm{x}_{\mathrm{n}}=\frac{\mathrm{x}_{\mathrm{n}+1}}{\mathrm{x}_{\mathrm{n}}}$, for uma $\mathrm{PG}(\mathrm{k}-1)$.

Com o propósito de simplificar notações, vamos fixar o seguinte; $\mathrm{q}_{0}=\mathrm{Q}^{0} \mathrm{x}_{1}, \mathrm{q}_{1}=\mathrm{Q}^{1} \mathrm{x}_{1}, \mathrm{q}_{2}=$ $\mathrm{Q}^{2} \mathrm{x}_{1}, \cdots, \mathrm{q}_{\mathrm{k}}=\mathrm{Q}^{\mathrm{k}} \mathrm{x}_{1}$. Veja que $\mathrm{q}_{0}$ é o primeiro termo da sequência original, $\mathrm{q}_{1}$ é o primeiro termo da sequência de quocientes de ordem 1 ; enfim, $\mathrm{q}_{\mathrm{k}}$ é o primeiro termo da sequência de quocientes de ordem $\mathrm{k}$.

A fórmula do n-ésimo termo e também a do produto dos n primeiros termos de uma PG(k) serão estabelecidas no seguinte teorema.

Teorema 4. Seja $\left(\mathrm{x}_{1}, \mathrm{x}_{2}, \ldots, \mathrm{x}_{\mathrm{n}}, \ldots\right)$ é uma Progressão Geométrica de ordem $\mathrm{k}>1$. O n-ésimo termo é determinado pela seguinte fórmula:

$$
\mathrm{x}_{\mathrm{n}}=\mathrm{q}_{0}^{\left(\begin{array}{c}
\mathrm{n}-1 \\
0
\end{array}\right)} \cdot \mathrm{q}_{1}^{\left(\begin{array}{c}
\mathrm{n}-1 \\
1
\end{array}\right)} \cdot \mathrm{q}_{2}^{\left(\begin{array}{c}
\mathrm{n}-1 \\
2
\end{array}\right)} \cdots \mathrm{q}_{\mathrm{k}}^{\left(\begin{array}{c}
\mathrm{n}-1 \\
\mathrm{k}
\end{array}\right)},
$$

e o produto de seus n primeiros termos é determinado por

$$
\prod_{i=1}^{n} x_{i}=q_{0}^{\left(\begin{array}{c}
n \\
1
\end{array}\right)} \cdot q_{1}^{\left(\begin{array}{c}
n \\
2
\end{array}\right)} \cdot q_{2}^{\left(\begin{array}{c}
n \\
3
\end{array}\right)} \cdots q_{k}^{\left(\begin{array}{c}
n \\
k+1
\end{array}\right)} .
$$

Demonstração. Vamos fazer primeiramente a prova da fórmula (11), para isso usaremos a indução sobre k. Se $\mathrm{k}=2$, temos;

$$
x_{n}=q_{0}^{\left(\begin{array}{c}
n-1 \\
0
\end{array}\right)} \cdot q_{1}^{\left(\begin{array}{c}
n-1 \\
1
\end{array}\right)} \cdot q_{2}^{\left(\begin{array}{c}
n-1 \\
2
\end{array}\right)}
$$

que é fórmula do n-ésimo termo de uma $\mathrm{PG}(2)$, conforme a igualdade (7), lembrando que $\mathrm{q}_{0}=\mathrm{x}_{1}$ e $\mathrm{q}_{2}=\mathrm{q}$. Por hipótese de indução, suponhamos que a fórmula seja válida para $\mathrm{PG}(\mathrm{k}-1)$. Sendo $\left(\mathrm{x}_{1}, \mathrm{x}_{2}, \ldots, \mathrm{x}_{\mathrm{n}}, \ldots\right)$ uma $\mathrm{PG}(\mathrm{k})$, segue que a sequência de quocientes $\left(\mathrm{Q}^{1} \mathrm{x}_{1}, \mathrm{Q}^{1} \mathrm{x}_{2}, \ldots, \mathrm{Q}^{1} \mathrm{x}_{\mathrm{n}}, \ldots\right)$ é uma $\mathrm{PG}(\mathrm{k}-1)$. Assim, pela hipótese de indução segue que

$$
\mathrm{Q}^{1} \mathrm{x}_{\mathrm{n}}=\mathrm{q}_{1}^{\left(\begin{array}{c}
\mathrm{n}-1 \\
0
\end{array}\right)} \cdot \mathrm{q}_{2}^{\left(\begin{array}{c}
\mathrm{n}-1 \\
1
\end{array}\right)} \cdot \mathrm{q}_{3}^{\left(\begin{array}{c}
\mathrm{n}-1 \\
2
\end{array}\right)} \cdots \mathrm{q}_{\mathrm{k}}^{\left(\begin{array}{c}
\mathrm{n}-1 \\
\mathrm{k}-1
\end{array}\right)} .
$$

Agora, levando em conta o Lema 1, a Observação 1, bem como essa ultima igualdade, obtemos;

$$
\begin{aligned}
\mathrm{x}_{\mathrm{n}} & =\mathrm{x}_{1} \cdot \frac{\mathrm{x}_{2}}{\mathrm{x}_{1}} \cdot \frac{\mathrm{x}_{3}}{\mathrm{x}_{2}} \cdots \frac{\mathrm{x}_{\mathrm{n}}}{\mathrm{x}_{\mathrm{n}-1}} \\
& =\mathrm{x}_{1} \cdot \mathrm{Q}^{1} \mathrm{x}_{1} \cdot \mathrm{Q}^{1} \mathrm{x}_{2} \cdot \mathrm{Q}^{1} \mathrm{x}_{3} \cdots \mathrm{Q}^{1} \mathrm{x}_{\mathrm{n}-1} \\
& =\mathrm{x}_{1} \cdot \prod_{\mathrm{i}=1}^{\mathrm{n}-1} \mathrm{Q}^{1} \mathrm{x}_{\mathrm{i}} \\
& =\mathrm{x}_{1} \cdot \mathrm{q}_{1}^{\sum_{\mathrm{i}=1}^{\mathrm{n}-1}\left(\begin{array}{c}
\mathrm{i}-1 \\
0
\end{array}\right)} \cdot \mathrm{q}_{2}^{\sum_{\mathrm{i}=1}^{\mathrm{n}-1}\left(\begin{array}{c}
\mathrm{i}-1 \\
1
\end{array}\right)} \cdot \mathrm{q}_{3}^{\sum_{\mathrm{i}=1}^{\mathrm{n}-1}\left(\begin{array}{c}
\mathrm{i}-1 \\
2
\end{array}\right)} \cdots \mathrm{q}_{\mathrm{k}}^{\sum_{\mathrm{i}=1}^{\mathrm{n}-1}\left(\begin{array}{c}
\mathrm{i}-1 \\
\mathrm{k}-1
\end{array}\right)} \\
& =\mathrm{q}_{0}^{\left(\begin{array}{c}
\mathrm{n}-1 \\
0
\end{array}\right)} \cdot \mathrm{q}_{1}^{\left(\begin{array}{c}
\mathrm{n}-1 \\
1
\end{array}\right)} \cdot \mathrm{q}_{2}^{\left(\begin{array}{c}
\mathrm{n}-1 \\
2
\end{array}\right)} \cdots \mathrm{q}_{\mathrm{k}}^{\left(\begin{array}{c}
\mathrm{n}-1 \\
\mathrm{k}
\end{array}\right)} .
\end{aligned}
$$


Nessa última igualdade levamos em conta o fato de que $\mathrm{q}_{0}=\mathrm{x}_{1}$ e $\mathrm{x}_{1}=\mathrm{x}_{1}^{\left(\begin{array}{c}\mathrm{n}-1 \\ 0\end{array}\right)}$.

A fim de provar a fórmula (12) utilizaremos a fórmula (11), o Lema 1 e a Observação 1. Assim, o produto dos n-primeiros termos de uma $\mathrm{PG}(\mathrm{k})$ é estabelecido da seguinte forma;

$$
\begin{aligned}
\prod_{i=1}^{n} x_{i} & =q_{0}^{\sum_{i=1}^{n}\left(\begin{array}{c}
n-1 \\
0
\end{array}\right)} \cdot q_{1}^{\sum_{i=1}^{n}\left(\begin{array}{c}
n-1 \\
1
\end{array}\right)} \cdot q_{2}^{\sum_{i=1}^{n}\left(\begin{array}{c}
n-1 \\
2
\end{array}\right)} \cdots q_{k}^{\sum_{i=1}^{n}\left(\begin{array}{c}
n-1 \\
k
\end{array}\right)} \\
& =q_{0}^{\left(\begin{array}{c}
n \\
1
\end{array}\right)} \cdot q_{1}^{\left(\begin{array}{c}
n \\
2
\end{array}\right)} \cdot q_{2}^{\left(\begin{array}{c}
n \\
2
\end{array}\right)} \cdots q_{k}^{\left(\begin{array}{c}
n \\
k+1
\end{array}\right)} .
\end{aligned}
$$

Mostraremos agora que a função de tipo exponencial $\mathrm{f}(\mathrm{x})=\mathrm{ba}^{\mathrm{x}}$, transforma PA(k) em PG(k).

Teorema 5. Sejam $\left(\mathrm{x}_{1}, \mathrm{x}_{2}, \ldots, \mathrm{x}_{\mathrm{n}}, \ldots\right)$ uma $P A(\mathrm{k})$ e $\mathrm{f}(\mathrm{x})=\mathrm{ba}^{\mathrm{x}}$ uma função do tipo exponencial. Então, a sequência $\left(\mathrm{f}\left(\mathrm{x}_{1}\right), \mathrm{f}\left(\mathrm{x}_{2}\right), \ldots, \mathrm{f}\left(\mathrm{x}_{\mathrm{n}}\right), \ldots\right)$ é uma $P G(\mathrm{k})$.

Demonstração. Faremos a demonstração por indução sobre k. Para k = 1 o resultado segue do Teorema 1. Suponhamos agora que o resultado seja válido para $\mathrm{k}=\mathrm{m}$, com $\mathrm{m} \geqslant 1$, provaremos que o resultado é válido para $\mathrm{k}=\mathrm{m}+1$.

Sendo $\left(\mathrm{x}_{1}, \mathrm{x}_{2}, \ldots, \mathrm{x}_{\mathrm{n}}, \ldots\right)$ uma $\mathrm{PA}(\mathrm{m}+1)$, segue que a sequência de diferenças $\left(\mathrm{d}_{1}, \mathrm{~d}_{2}, \ldots, \mathrm{d}_{\mathrm{n}}, \ldots\right)$, onde $d_{n}=x_{n+1}-x_{n}$, é uma PA(m). Agora, pela hipótese de indução, segue que $\left(a^{d_{1}}, a^{d_{2}}, \ldots, a^{d_{n}}, \ldots\right)$ é uma $\mathrm{PG}(\mathrm{m})$, pois é a imagem de $\left(\mathrm{d}_{1}, \mathrm{~d}_{2}, \ldots, \mathrm{d}_{\mathrm{n}}, \ldots\right)$ pela função exponencial $\mathrm{g}(\mathrm{x})=\mathrm{a}^{\mathrm{x}}$. Por fim, veja que $\frac{f\left(x_{n+1}\right)}{f\left(x_{n}\right)}=a^{x_{n+1}-x_{n}}=a^{d_{n}}$, garantindo que $\left(f\left(x_{1}\right), f\left(x_{2}\right), \ldots, f\left(x_{n}\right), \ldots\right)$ é uma $P G(m+1)$, finalizando a demonstração.

Para finalizar, é importante fazermos uma observação com respeito à recíproca do teorema anterior, isto é, se existe uma função que transforma uma PG(k) em uma PA(k). Pelo menos quando consideramos uma $\mathrm{PG}(\mathrm{k})$ com termos positivos isto é verdadeiro, considerando uma função logarítmica, como pode ser visto no teorema abaixo.

Teorema 6. Sejam $\left(\mathrm{x}_{1}, \mathrm{x}_{2}, \ldots, \mathrm{x}_{\mathrm{n}}, \ldots\right)$ uma $P G(\mathrm{k})$ de termos positivos e $\mathrm{g}(\mathrm{x})=\log _{\mathrm{a}}(\mathrm{x})$, com a $>0$ e a $\neq 1$, uma função logarítmica. Então, a sequência $\left(\mathrm{g}\left(\mathrm{x}_{1}\right), \mathrm{g}\left(\mathrm{x}_{2}\right), \ldots, \mathrm{g}\left(\mathrm{x}_{\mathrm{n}}\right), \ldots\right)$ é uma $P A(\mathrm{k})$.

Demonstração. Faremos a demonstração por indução sobre k. Veja que o resultado é válido para $\mathrm{k}=1$, pois, neste caso, $\left(\mathrm{x}_{1}, \mathrm{x}_{2}, \ldots, \mathrm{x}_{\mathrm{n}}, \ldots\right)$ é uma $\mathrm{PG}$, cuja razão será denotada por q. Note que $\log _{\mathrm{a}}\left(\mathrm{x}_{\mathrm{n}+1}\right)-\log _{\mathrm{a}}\left(\mathrm{x}_{\mathrm{n}}\right)=\log _{\mathrm{a}}\left(\frac{\mathrm{x}_{\mathrm{n}+1}}{\mathrm{x}_{\mathrm{n}}}\right)=\log _{\mathrm{a}}(\mathrm{q})$, para qualquer que seja o número natural $\mathrm{n}$. Isso mostra que $\left(\mathrm{g}\left(\mathrm{x}_{1}\right), \mathrm{g}\left(\mathrm{x}_{2}\right), \ldots, \mathrm{g}\left(\mathrm{x}_{\mathrm{n}}\right), \ldots\right)$ é uma PA de razão $\log _{\mathrm{a}}(\mathrm{q})$.

Suponhamos agora que o resultado seja válido para $\mathrm{k}=\mathrm{m}$ e $\mathrm{m} \geq 1$. Sendo $\left(\mathrm{x}_{1}, \mathrm{x}_{2}, \ldots, \mathrm{x}_{\mathrm{n}}, \ldots\right)$ uma $P G(m+1)$ segue que a sequência de quocientes $\left(Q_{1}, Q_{2}, \ldots, Q_{n}, \ldots\right)$, com $Q_{n}=\frac{x_{n+1}}{x_{n}}$, é uma $P G(m)$. Daí, pela hipótese de indução segue que $\left(\mathrm{g}\left(\mathrm{Q}_{1}\right), \mathrm{g}\left(\mathrm{Q}_{2}\right), \ldots, \mathrm{g}\left(\mathrm{Q}_{\mathrm{n}}\right), \ldots\right)$ é uma $\mathrm{PA}(\mathrm{m})$. Notando que $\mathrm{g}\left(\mathrm{x}_{\mathrm{n}+1}\right)-\mathrm{g}\left(\mathrm{x}_{\mathrm{n}}\right)=\log _{\mathrm{a}}\left(\frac{\mathrm{x}_{\mathrm{n}+1}}{\mathrm{x}_{\mathrm{n}}}\right)=\mathrm{g}\left(\mathrm{Q}_{\mathrm{n}}\right)$, garantimos que $\left(\mathrm{g}\left(\mathrm{x}_{1}\right), \mathrm{g}\left(\mathrm{x}_{2}\right), \ldots, \mathrm{g}\left(\mathrm{x}_{\mathrm{n}}\right), \ldots\right)$ é uma PA $(\mathrm{m}+1)$, como queríamos demonstrar. 


\section{Referências}

[1] Carvalho, P.C P., Morgado, A. C O., Fernandez P., Pitombeira, J. B. Análise Combinatória e Probabilidade. Rio de Janeiro, SBM, 2000.

[2] Hefez, A. Curso de Álgebra, Vol. 1. $3^{\text {a }}$ ed. Coleção Matemática Universitária; Rio de Janeiro, 2002.

[3] Lima, E. L. Números e Funções. Coleção Profmat; Rio de Janeiro, SBM, 2013.

[4] Lima, W. A. F. Progressões Aritméticas de Ordem Superior: uma proposta de abordagem no Ensino Médio. Dissertação (Profmat-UEM), Maringá-PR, 2015.

[5] Lopes, F. H. O ensino de Progressão Geométrica de segunda ordem no Ensino Médio. Dissertação (Profmat-Unesp), São José do Rio Preto - SP, 2017.

[6] Morgado, A. C.; Carvalho, P. C. P. Matemática Discreta. Coleção Profmat; Rio de Janeiro, SBM, 2013.

[7] Nobre, J. F. F. Progressões Aritméticas: abordando as ordens superiores. Dissertação (ProfmatUFT), Palmas-TO, 2018.

[8] Nobre, J. F. F.; Rocha, R. A. "Progressões Aritméticas de Ordem Superior". Revista Professor de Matemática Online, v.5, n.1, 2018.

Ruikson S. O. Nunes

Universidade Federal de Mato Grosso-UFMT Instituto de Ciências Exatas e da Terra-Icet Departamento de Matemática <ruiksonsillas@hotmail.com>

Juliano Sanquite Gomes Universidade Federal de Mato Grosso-UFMT Instituto de Ciências Exatas e da Terra-Icet Departamento de Matemática <san27g@gmail.com>

Recebido: 14/02/2019 Publicado: 19/11/2020 